\title{
Spectral and Photophysical Studies of Poly[2,6-(1,5-dioctylnaphthalene)]thiophenes
}

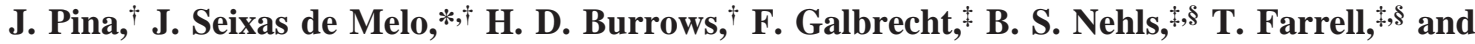 \\ U. Scherf: \\ Department of Chemistry, University of Coimbra, Rua Larga, 3004-535 Coimbra, Portugal, and \\ Makromolekulare Chemie, Bergische Universität Wuppertal, D-42097 Wuppertal, Germany
}

Received: January 26, 2007; In Final Form: March 8, 2007

\begin{abstract}
A complete spectroscopic and photophysical study of three alternating naphthalene- $\alpha$-thiophene copolymers was undertaken in solution (room and low temperature) and in the solid state (thin films in a Zeonex matrix). The study comprises absorption, emission, and triplet-triplet spectra together with quantitative measurements of quantum yield (fluorescence, intersystem-crossing, internal conversion, and singlet oxygen formation) lifetimes and singlet and triplet energies. The overall data allow the determination of the rate constants for all the decay processes. Comparison between the behavior of analogous 1-naphthyl(oligo)thiophenes and the 2,6-naphthalene(oligo)thiophene copolymers allows several important observations. First, the polymers display higher fluorescence quantum yields and lower $S_{1} \sim \sim T_{1}$ intersystem-crossing yields than the oligomers. This can be attributed to the presence of the 1,5-dioctyloxynaphthalene groups in the copolymers leading to a more rigid polymer backbone, which decreases radiationless deactivation and increases the radiative efficiency. Second, the singlet and triplet energies are significantly lower in the polymers than with the corresponding oligomers. This implies a lower HOMO-LUMO energy difference in the polymers due to an extended $\pi$-delocalization. Third, the singlet-to-triplet $\left(\mathrm{S}_{1}-\mathrm{T}_{1}\right)$ energy splitting is higher in the oligomers than with the polymers, even though the former display higher intersystem-crossing yields. It is suggested that this may result from intersystem-crossing in the oligomers involving significant charge-transfer (CT) character (spinorbit coupling is mediated by CT mixing involving the singlet and triplet states in matrix elements of the type $\left.\left\langle{ }^{1} \Psi_{\mathrm{CT}}\left|\mathrm{H}^{\prime}\right|^{3} \Psi_{1}\right\rangle\right)$ of the relevant excited states but that is less important with the polymers. We believe that this may be relevant to understanding the nature of CT states in conjugated copolymers.
\end{abstract}

\section{Introduction}

Polythiophenes have emerged as one of the most versatile classes of $\pi$-conjugated systems included in the broad area of conjugated organic polymers. ${ }^{1-3}$ Their unique properties are demonstrated by their high-chemical stability and by the possibility of tuning the spectral properties and photophysical behavior over a wide range through modification of their chemical structure (e.g., by incorporation of lateral groups attached to the polythiophene backbone).$^{4-6}$ An alternative approach is to incorporate the thiophene moieties in copolymers. In a previous study, we reported the effect of terminal naphthalene substitution at the $\alpha$-position on the photophysical properties of oligothiophenes. ${ }^{7}$ Here, alternating copolymers of 1,5-dioctyloxynaphthalene linked in the 2,6 position to three thiophene oligomers ( $\alpha 1, \alpha 2$, and trans-dithienylvinylene) were synthesized with the objective of improving their luminescence efficiency, charge transport properties, and in understanding the factors that affect the nonradiative decay, in particular through intersystem-crossing, in these systems. Copolymers containing at least two different conjugated segments are expected to exhibit novel electronic, optoelectronic, and optical properties that are

* To whom correspondence should be addressed. E-mail: sseixas@ci.uc.pt. Fax: 351239827703 .

University of Coimbra.

$\doteqdot$ Bergische Universität Wuppertal.

$\S$ Present addresses. (T.F.) GE Plastics, Plasticslaan 1, P.O. Box 117, 4600 AC Bergen op Zoom, The Netherlands. (B.S.N.) BASF, GKT/F-B001, D-67056 Ludwigshafen, Germany. not found in the individual homopolymers. Also, the electronic properties of the conjugated polymers are likely to be highly dependent on both the nature of the active building blocks and the way in which they are linked.

It is known that the highest occupied molecular orbitallowest unoccupied molecular orbital (HOMO-LUMO) energy gap of $\pi$-conjugated systems containing heteroaromatic units depends on various structural factors such as bond length alternation, planarity, aromatic resonance energy, and electronic effects promoted by side chain substituents. ${ }^{8}$ In the present study, the spectral and photophysical properties of three of these alternating copolymers were investigated in solution (room and low temperature) and in the solid state (thin films). With one of the copolymers, samples of two different molecular weights and polydispersities were studied to see whether this has any effects on either photophysical or film-forming properties.

\section{Experimental Methods}

The synthesis of the copolymers was done in microwaveassisted process as reported for DONp $2 . .^{9}$ Detailed synthesis procedures and characterization data for all the copolymers can be found in the Supporting Information. All the solvents used were of spectroscopic or equivalent grade. For the absorption and emission experiments in toluene, the concentrations of the solutions ranged from $1 \times 10^{-5}$ to $10^{-6} \mathrm{M}$ (in terms of repeat units).

Absorption and fluorescence spectra were recorded on Shimadzu UV-2100 and Horiba-Jobin-Ivon SPEX Fluorog 3-22 
spectrometers respectively. The fluorescence spectra were corrected for the wavelength response of the system.

The fluorescence quantum yields were measured using quinquethiophene $\left(\phi_{\mathrm{F}}=0.33\right.$ in methylcyclohexane, $\left.\mathrm{MCH}\right)$ as standard. ${ }^{10}$

The fluorescence quantum yields at $77 \mathrm{~K}$ were obtained by comparison with the spectrum at $293 \mathrm{~K}$ run under the same experimental conditions, and the $\phi_{\mathrm{F}}$ value was obtained by assuming a shrinkage of the solvent volume $(V)$ upon cooling given by $V_{293 \mathrm{~K}} / V_{77 \mathrm{~K}}=0.8 .{ }^{11}$ The low-temperature experiments were performed in $\mathrm{MCH}$ instead of toluene (a better solvent for the polymers) because toluene does not form a good glass at low temperature, whereas with $\mathrm{MCH}$ perfect glasses were obtained upon cooling the room-temperature solution.

Fluorescence decays were measured using a home-built time correlated single photon counting (TCSPC) apparatus with an IBH NanoLED (373 or $460 \mathrm{~nm}$ ) excitation source, Jobin-Yvon excitation and emission monochromators, Philips XP2020Q photomultiplier, and Canberra instruments time-to-amplitude (TAC) and multi-channel analyzer (MCA). Alternate measurements $(1000 \mathrm{cpc})$ of the pulse profile at the excitation wavelength and the sample emission in solution were performed until $5 \times 10^{4}$ counts at the maximum were reached. Solid-state fluorescence decays were measured with samples in a HoribaJobin-Yvon integrating sphere. For these experiments, the pulse profile at the excitation wavelength was obtained by collecting the pulse with a sapphire blank disk inside the integrating sphere. In this way, it was possible to produce the pulse profile with the instrumental response as generated within the integrating sphere. This is seen to be different from the usual pulse profile (generated with a scattering compound such as Ludox or glycogen) and is reflected by the presence of a much longer tail (see Figure 3 in Results and Discussion). The fluorescence decays were analyzed using the modulating functions method of Striker with automatic correction for the photomultiplier "wavelength shift". ${ }^{12}$

The experimental setup used to obtain singlet-triplet depletion spectra and triplet state quantum yields consists of an Applied Photophysics laser flash photolysis apparatus pumped by a Nd:YAG laser (Spectra Physics). The detection system is at right angles to the excitation beam, and a pulsed $150 \mathrm{~W} \mathrm{Xe}$ lamp is used to analyze the transient absorption. The signal is fed into an HP digital analyzer and transferred to an IBM RISC computer in which the optical density (OD) at different wavelengths and different delays after flash are collected using the appropriate software (Applied Photophysics). Transient spectra were obtained by monitoring the OD change at intervals of $5-10 \mathrm{~nm}$ over the $300-850 \mathrm{~nm}$ range and averaging at least 10 decays at each wavelength. First-order kinetics were observed in all cases for the decay of the lowest triplet state. To avoid multiphoton and $\mathrm{T}-\mathrm{T}$ annihilation effects, special care was taken to have optically matched dilute solutions $(\mathrm{abs} \approx 0.2$ in a 10 $\mathrm{mm}$ square cell) and low laser energy $(\leq 2 \mathrm{~mJ})$ in determining the triplet yields.

The triplet molar absorption coefficients were obtained by the energy transfer method. ${ }^{13}$ The triplet state molar absorption coefficients were determined using biphenyl, $\epsilon_{\mathrm{T}}=27100 \mathrm{M}^{-1}$ $\mathrm{cm}^{-1}(360 \mathrm{~nm})$, or pyrene, $\epsilon_{\mathrm{T}}=20900 \mathrm{M}^{-1} \mathrm{~cm}^{-1}(420 \mathrm{~nm})$, as triplet energy donors. ${ }^{11}$ The concentrations for the compounds studied were $10^{-5} \mathrm{M}$ (in terms of repeat units), and they were dissolved in benzene or toluene solutions of biphenyl or pyrene $(10 \mathrm{mM})$. Before experiments, all solutions were degassed with argon for $\approx 30 \mathrm{~min}$ and were sealed. The triplet-triplet molar absorption coefficients were then determined at the analytical wavelength for triplet quantum yield determinations from eq $1^{13}$

$$
\frac{\epsilon_{\mathrm{TT}}^{\mathrm{D}}}{\epsilon_{\mathrm{TT}}^{\mathrm{A}}}=\frac{\Delta \mathrm{OD}^{\mathrm{D}}}{\Delta \mathrm{OD}^{\mathrm{A}}}
$$

where $\epsilon_{\mathrm{TT}}^{\mathrm{D}}$ and $\epsilon_{\mathrm{TT}}^{\mathrm{A}}$ are the triplet state molar absorption coefficients of donor and acceptor, respectively, $\Delta \mathrm{OD}^{\mathrm{D}}$ is the maximum absorbance from the transient triplet-triplet absorption spectra of the donor in the absence of acceptor, and $\Delta \mathrm{OD}^{\mathrm{A}}$ is the maximum absorbance of the acceptor triplet when both the donor and acceptor are present. For determination of $\Delta \mathrm{OD}^{\mathrm{A}}$, additional corrections were made for cases when the acceptor decay rate constant $\left(k_{3}\right)$ is not negligible. For this situation, eq 2 was applied ${ }^{13}$

$$
\Delta \mathrm{OD}_{\mathrm{obs}}^{\mathrm{A}}=\Delta \mathrm{OD}^{\mathrm{A}} \exp \left[-\frac{\ln k_{2} / k_{3}}{k_{2} / k_{3}-1}\right]
$$

where $k_{2}$ is the donor decay rate constant in the presence of acceptor and $\Delta \mathrm{OD}_{\mathrm{obs}}^{\mathrm{A}}$ is taken from the maximum observed in the triplet-singlet difference spectra of the acceptor in the presence of donor.

The intersystem-crossing yields for the compounds $\left(\phi_{\mathrm{T}}^{\mathrm{cp}}\right)$ were obtained by comparing the $\triangle \mathrm{OD}$ at $525 \mathrm{~nm}$ of benzene solutions optically matched (at the laser excitation wavelength) of benzophenone (standard) and of the compound using the equation ${ }^{7,14}$

$$
\phi_{\mathrm{T}}^{\mathrm{cp}}=\left(\frac{\epsilon_{\mathrm{TT}}^{\text {Benzophenone }}}{\epsilon_{\mathrm{TT}}^{\mathrm{cp}}}\right)\left(\frac{\Delta \mathrm{OD}_{\max }^{\mathrm{cp}}}{\Delta \mathrm{OD} \mathrm{D}_{\max }^{\text {Benzophenone }}}\right)\left(\phi_{\mathrm{T}}^{\text {Benzophenone }}\right)
$$

Triplet state absorption spectra were also characterized by pulse radiolysis using the Free Radical Research Facility, Daresbury, UK. Here, 200 ns to $2 \mu$ s high-energy electron pulses from a $12 \mathrm{MeV}$ linear accelerator were passed through solutions in a $2.5 \mathrm{~cm}$ optical path length quartz cuvette attached to a flow system, as has been described in detail elsewhere. ${ }^{15}$ All solutions were bubbled with argon for about 30 min before experiments. Spectra are comparable with those obtained by laser flash photolysis, except at longer wavelengths $(>700 \mathrm{~nm})$, in which those obtained by flash photolysis appear to be attenuated by the diminished response of the detector.

Room-temperature singlet oxygen phosphorescence was detected at $1270 \mathrm{~nm}$ using a Hamamatsu R5509-42 photomultiplier cooled to $193 \mathrm{~K}$ in a liquid nitrogen chamber (Products for Research model PC176TSCE-005) following laser excitation of aerated solutions at $355 \mathrm{~nm}(\mathrm{OD}$ at $355 \mathrm{~nm}=0.20)$ with an adapted Applied Photophysics flash kinetic spectrometer. The modification of the spectrometer involved the interposition of a Schott RG665 filter. A 600-line diffraction grating was used instead of the standard spectrometer one to extend spectral response to the infrared. The filter employed is essential to eliminate all the first harmonic contributions from the sensitizer emission in the $500-800 \mathrm{~nm}$ region from the infrared signal. $1 H$-Phenalen-1-one (perinaphthenone) in toluene $\left(\phi_{\Delta}=0.93\right)$ was used as standard. ${ }^{16}$

Thin films from the compounds were obtained with a desktop precision spin-coating system, Model P6700 Series from Speedline Technologies. The solid-state thin film from the samples was obtained by deposition of a few drops from a solution of the compounds onto a circular sapphire substrate (10 $\mathrm{mm}$ diameter) followed by spin-coating $(2500 \mathrm{rpm})$ in a nitrogen-saturated atmosphere (2 psi). The solutions for spin- 


\section{SCHEME 1}
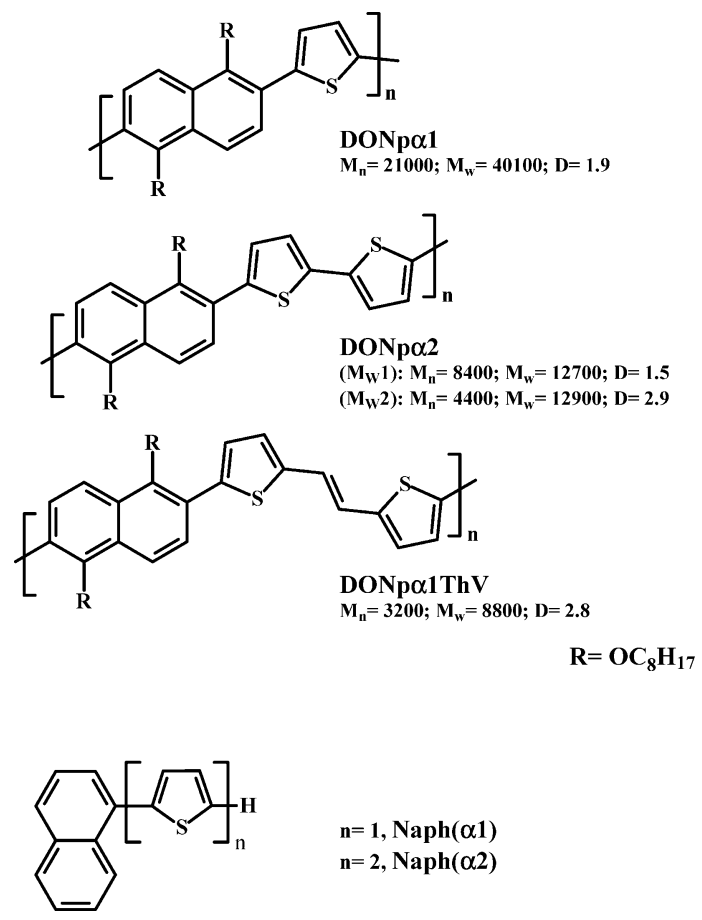

$\mathrm{n}=1, \operatorname{Naph}(\alpha 1)$

$\mathrm{n}=2, \operatorname{Naph}(\alpha 2)$

coating were prepared by adding $2 \mathrm{mg}$ of the samples to $15 \mathrm{mg}$ of Zeonex in $200 \mu \mathrm{L}$ toluene solution with stirring at $40{ }^{\circ} \mathrm{C}$ for $30 \mathrm{~min}$.

In one case, a polymer film was prepared without Zeonex by spin-coating. However, no significant differences in the emission and absorption spectra (see Supporting Information) were observed between this and the blend made of the copolymer with the polyolefin Zeonex. Because of the limited quantities of the copolymers available, the latter technique was chosen for detailed photophysical studies on films.

The fluorescence emission spectra of the thin films were obtained with a Horiba-Jobin-Yvon integrating sphere. The solid-state photoluminescence quantum yields in thin films were obtained with this integrating sphere, using the method described elsewhere. ${ }^{17}$

\section{Results}

The structures, number average $\left(M_{n}\right)$, weight average $\left(M_{\mathrm{W}}\right)$ molecular weights, polydispersity $\left(D=M_{\mathrm{W}} / M_{n}\right)$ and acronyms of the samples studied are presented in Scheme 1. The structures of two 1-naphthyl(oligo)thiophenes, $\operatorname{Naph}(\alpha n)$, with one naphthalene unit and one $[\operatorname{Naph}(\alpha 1)]$ or two $[\operatorname{Naph}(\alpha 2)]$ thienyl units, which have previously been investigated ${ }^{7}$ and are used for comparison purposes, are also depicted in Scheme 1. The systems in this study are alternating copolymers of 1,5dioctyloxynaphthalene linked in the 2,6-position to different thiophene oligomers ( $\alpha 1, \alpha 2$, and trans-dithienylvinylene). In Figure 1, the solution absorption spectra at room (293 K) and low temperature (given by the fluorescence excitation spectra) and in the solid state (thin films in Zeonex) are shown. With these alternating copolymers, the room-temperature absorption spectra are devoid of vibrational resolution in contrast to what is generally observed with aromatic hydrocarbons, such as naphthalene in which absorption bands show highly resolved vibronic structure.

The absorption spectra of the copolymers studied strongly resemble those reported for the related 1-naphthyl(oligo)thiophenes, ${ }^{7}$ except for a red-shift in the wavelength maxima,

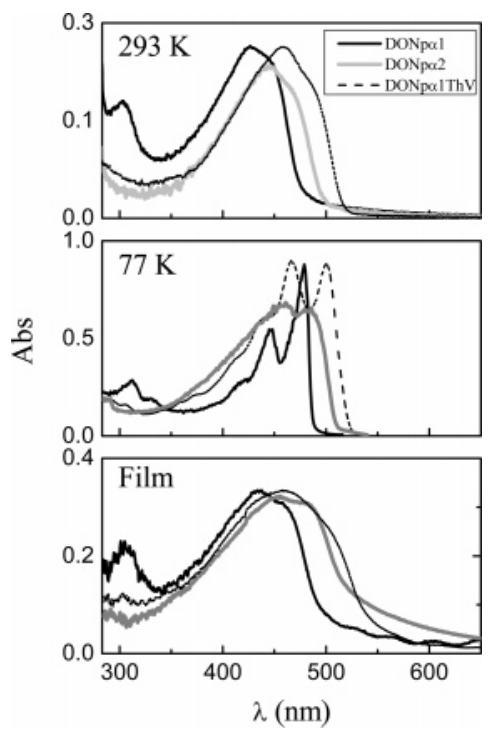

Figure 1. Absorption spectra for the copolymers in a toluene solution at room temperature $(293 \mathrm{~K})$, at low temperature (determined as excitation spectra), and in thin films. The DONpa2 polymer presented is labeled with $M_{\mathrm{W}} 1$ (see Table 1 , Scheme 1 , and text for further details).

which increases with the number of thiophene units and with the introduction of the trans-dithienylvinylene group. It should be stressed that the band due to naphthalene moiety could not be detected, indicating that naphthalene is involved with the oligothiophene unit in the overall conjugation segment.

On decreasing the temperature, a red-shift of the absorption was observed with shifts in the absorption maxima ranging from $\approx 16 \mathrm{~nm}(\mathrm{DONp} \alpha 2)$ to $\approx 53 \mathrm{~nm}(\mathrm{DONp} \alpha 1)$ on going from 293 to $77 \mathrm{~K}$. Similar behavior was observed for the solid-state absorption band in a Zeonex matrix (relative to solution at 293 $\mathrm{K})$, although, with the exception of the copolymer containing the trans-dithienylvinylene substituent (DONpa1ThV), the shifts were smaller (see Table 1 and Figure 1).

Figure 2 shows fluorescence emission spectra of the copolymers at room temperature $(293 \mathrm{~K})$, low temperature $(77 \mathrm{~K})$, and in films. In contrast to the absorption spectra, the roomtemperature fluorescence emission bands present vibrational structure. However, again there is no evidence for naphthalene emission, supporting the idea that both naphthalene and oligothiophenes are involved in the conjugation segment.

On going from 293 to $77 \mathrm{~K}$, a red-shift in the emission band was observed with negligible spectral narrowing in the emission structure (Figure 2). The exception is observed with DONp $\alpha 1$ in which a pronounced change in the vibronic bandwidth is seen. If it is assumed, as is common with conjugated polymers, that a large part of the spectral broadening is due to the presence of different conformations, this marked decrease in the fluorescence bandwidth of DONpa1 on going from 293 to $77 \mathrm{~K}$ suggests that as the temperature is lowered there is a smaller variation in the planarity among the potential existing conformers and that this is more pronounced with the shorter copolymer. Moreover it also suggests that in the case of the naphthalenethiophene copolymer DONp $\alpha 1$ the $S_{1}$ state has less of the quinoidal-like structure, which is characteristic of the $S_{1}$ state in the $\alpha$-oligothiophenes ${ }^{10}$ and $\operatorname{Naph}(\alpha n)^{7}$ oligomers.

In thin films made of blends of the copolymers with the polyolefin, Zeonex, a loss of structure of the emission spectra occurred with concomitant broadening, red-shift, and decrease in quantum yield. These results strongly suggest aggregation.

Fluorescence lifetimes $\left(\tau_{\mathrm{F}}\right)$ were also obtained in solution and in the solid state and (within our time-resolution, $\approx 150 \mathrm{ps}$ ) were 
TABLE 1: Spectroscopic Data for the Studied Copolymers in Toluene at Room Temperature (293 K), Low Temperature (77 $\mathrm{K})$, and in the Solid State (Thin Films) ${ }^{a}$

\begin{tabular}{|c|c|c|c|c|c|c|c|c|c|}
\hline compound & $\begin{array}{c}\lambda_{\max }^{\mathrm{Abs}} \\
(\mathrm{nm}) \\
293 \mathrm{~K}\end{array}$ & $\begin{array}{l}\lambda_{\max }^{\mathrm{Abs}} \\
(\mathrm{nm}) \\
77 \mathrm{~K}\end{array}$ & $\begin{array}{l}\lambda_{\max }^{\mathrm{Abs}} \\
(\mathrm{nm}) \\
\text { film }\end{array}$ & $\begin{array}{c}\lambda_{\max }^{\text {Fluo }} \\
(\mathrm{nm}) \\
293 \mathrm{~K}\end{array}$ & $\begin{array}{l}\lambda_{\max }^{\text {Fluo }} \\
(\mathrm{nm}) \\
77 \mathrm{~K}\end{array}$ & $\begin{array}{l}\lambda_{\max }^{\text {Fluo }} \\
(\mathrm{nm}) \\
\text { film }\end{array}$ & $\begin{array}{c}\lambda \lambda_{\max }^{T_{1} \rightarrow T_{n}} \\
(\mathrm{~nm})\end{array}$ & $\begin{array}{c}\epsilon_{\mathbf{T T}} \\
\left(\mathrm{M}^{-1} \mathrm{~cm}^{-1}\right)\end{array}$ & $\begin{array}{c}\Delta_{\mathrm{Ss}} \\
\left(\mathrm{cm}^{-1}\right) \\
293 \mathrm{~K}\end{array}$ \\
\hline $\mathrm{DONp} \alpha 1$ & $302, \underline{426}$ & $\begin{array}{c}312,330,418 \\
445,479\end{array}$ & $305, \underline{434}$ & $\underline{470}, 499,529$ & $\underline{481}, 496,518$ & $470, \underline{502}$ & 750 & 13850 & 2198 \\
\hline $\operatorname{DONp} \alpha 2\left(M_{\mathrm{w}} 1\right)$ & 444 & $\underline{460}, 48 \overline{3}$ & 454 & $494,528,567$ & $\frac{500,534,577,}{628}$ & 548 & 690 & 29780 & 2280 \\
\hline $\mathrm{DONp} \alpha 2\left(M_{\mathrm{w}} 2\right)$ & 440 & 460 & 445 & $494,528,567$ & $\frac{500,534,577,}{628}$ & $\underline{535}, 571$ & 670 & 27510 & 2484 \\
\hline DONp $\alpha 1 \mathrm{ThV}$ & 458 & $\underline{466}, 500$ & 458 & $\underline{511}, 548,590$ & $\frac{518,557,602}{657}$ & 550 & 630 & 45000 & 2265 \\
\hline $\operatorname{Naph}(\alpha 1)^{b}$ & 297 & 307 & & 378 & 348,365 & & $340,470^{c}$ & & 7215 \\
\hline $\operatorname{Naph}(\alpha 2)^{b}$ & 335 & 347 & & 435 & $399, \underline{419}$ & & $405,{\underline{555^{c}}}^{-}$ & 33913 & 6862 \\
\hline
\end{tabular}

${ }^{a}$ The underlined wavelengths is the band maximum. ${ }^{b}$ Data in MCH taken from ref $7 .{ }^{c}$ Data in benzene taken from ref 7.

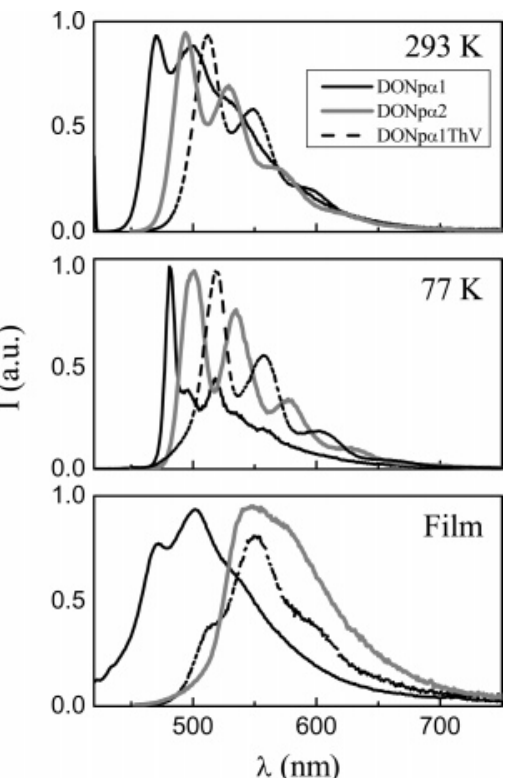

Figure 2. Fluorescence emission spectra for the copolymers in solution at room temperature $(293 \mathrm{~K})$, at low temperature $(77 \mathrm{~K})$, and in Zeonex films. The DONpa2 polymer presented is labeled with $M_{\mathrm{W}} 1$ (see Table 1 , Scheme 1, and text for further details).

seen to be single exponential; see Table 2 and Figure 3 for an illustrative example of a decay (in solution and in film).

Transient triplet-triplet absorption spectra for the copolymers were obtained in toluene solution at $293 \mathrm{~K}$ by laser flash photolysis (Figure 4). In addition to ground-state depletion, the spectra show an intense absorption with bands between 500 and $800 \mathrm{~nm}$ (see Table 1 and Figure 4). The transient triplet-triplet absorption spectra for these samples are broad (see Figure 4), suggesting some delocalization of the triplet excited-state resulting from an effective conjugation along the copolymer backbone.

Both the spectra and the energies of the triplet state of these compounds were also investigated by the pulse radiolysis energy transfer method in benzene. Here, the triplet states of conjugated polymers or copolymers $(\mathrm{P})$ could be selectively produced by energy transfer from appropriate sensitizers (S) following pulse radiolysis of benzene $(\mathrm{Bz})$ solutions as illustrated in Scheme $2 .{ }^{18,19}$

This reaction scheme is subject to the kinetically demanded concentration ratio $[\mathrm{Bz}] \gg[\mathrm{S}] \gg[\mathrm{P}]$. Biphenyl $(10 \mathrm{mM})$ was used as sensitizer. Similar transient absorption bands were observed as to those seen in the laser flash photolysis experiments (Figure 4), except at longer wavelengths for reasons discussed in Experimental Methods.
Singlet oxygen formation quantum yields $\left(\phi_{\Delta}\right)$ were obtained following photolysis of aerated toluene solutions of the copolymers. The $\phi_{\Delta}$ values were determined by plotting the initial singlet oxygen phosphorescence intensity $(1270 \mathrm{~nm})$ as a function of the laser dose and comparing the slope with that obtained upon sensitization with $1 \mathrm{H}$-phenalen-1-one in toluene as the standard. The values obtained are presented in Table 2.

\section{Discussion}

The photophysical parameters obtained in solution and in the solid state are presented in Table 2 , and the rate constants for the radiative $\left(k_{\mathrm{F}}\right)$ and radiationless processes $\left(k_{\mathrm{NR}}=k_{\mathrm{IC}}+k_{\mathrm{ISC}}\right)$ in Table 3. The general trend found for the behavior in solution shows that for all the copolymers studied (see Table 2) the radiationless processes (i.e., the internal conversion $\left(\phi_{\mathrm{IC}}\right)$ and $\mathrm{S}_{1} \sim \sim \mathrm{T}_{1}$ intersystem-crossing yield $\left.\left(\phi_{\mathrm{T}}\right)\right)$ are more important than the radiative processes in the excited-state deactivation. Although it is possible that some charge separation occurs in the solid-state with these copolymers, the reasonable photostability and lack of long-lived species suggests that if this occurs it must be followed by very rapid charge recombination leading to either excited triplet state or ground state, as suggested elsewhere. ${ }^{20}$ The fluorescence quantum yield $\left(\phi_{\mathrm{F}}\right)$ increases from the polymer with one thiophene to the polymer with two thiophenes (see DONpa1 and DONpo2 in Table 2). This is in contrast with the behavior of alternating fluorene-thiophene copolymers $^{21}$ in which a slight decrease in $\phi_{\mathrm{F}}$ is seen on going from the thiophene to the terthiophene copolymer.

In comparison with the respective 1-naphthyl(oligo)thiophene counterparts (Naph $\alpha 1$ and Naph $\alpha 2),{ }^{7}$ the naphthalene-thiophene copolymers were found to display higher fluorescence quantum yields and lower $\mathrm{S}_{1} \sim \sim \mathrm{T}_{1}$ intersystem-crossing yields (see Table 2). This could be attributed to the additional 1,5dioctyloxy groups in the naphthalene moieties that lead the polymer to display a decrease of the radiationless deactivation pathways and an increase of the radiative (fluorescence) efficiency when compared to the oligomers. ${ }^{7,22,23}$

Introduction of the trans-dithienylvinylene group (DONp $\alpha 1 \mathrm{ThV}$ ) in the polymer backbone more than doubles the $\phi_{\mathrm{F}}$ value relative to DONp $\alpha 1$ and increases it by about $50 \%$ relative to DONpa2 (see Table 2). The structural modification induced by the introduction of double bonds of trans configuration between thiophene rings may have the effect of planarization of the ground state and the increase of the overall aromaticity of the $\pi$-conjugated system thus decreasing the HOMO-LUMO energy difference. ${ }^{24}$ While $k_{\mathrm{F}}$ in DONp $\alpha 1 \mathrm{ThV}$ is significantly greater than in DONp $\alpha 1$, it is comparable to DONpa2. The biggest change is associated with the decreased 
TABLE 2: Photophysical Parameters for the Studied Copolymers in Toluene at Room Temperature (293 K), Low Temperature (77 K), and in Thin Films

\begin{tabular}{|c|c|c|c|c|c|c|c|c|c|c|c|c|}
\hline compound & $\begin{array}{c}\phi_{\mathbf{F}} \\
293 \mathrm{~K}^{g}\end{array}$ & $\begin{array}{c}\tau_{\mathrm{F}}(\mathrm{ns}) \\
293 \mathrm{~K}\end{array}$ & $\begin{array}{c}\phi_{\mathbf{F}} \\
77 \mathrm{~K}^{a, g}\end{array}$ & $\begin{array}{c}\phi_{\mathbf{F}} \\
\text { film }\end{array}$ & $\begin{array}{c}\tau_{\mathbf{F}}(\mathrm{ns}) \\
\text { film }^{g}\end{array}$ & $\begin{array}{c}\phi_{\mathbf{I C}} \\
293 \mathrm{~K}^{f}\end{array}$ & $\begin{array}{c}\phi_{\mathbf{T}} \\
293 \mathrm{~K}^{h}\end{array}$ & $\begin{array}{c}\phi_{\Delta} \\
293 \mathrm{~K}\end{array}$ & $\begin{array}{l}\tau_{\mathbf{T}}(\mu \mathrm{s}) \\
293 \mathrm{~K}\end{array}$ & $\begin{array}{l}\text { Triplet } \\
\text { energy } \\
(\mathrm{eV})^{b}\end{array}$ & $\begin{array}{c}\text { Singlet } \\
\text { energy } \\
(\mathrm{eV})^{e}\end{array}$ & $\begin{array}{c}\Delta E_{\mathrm{S}_{1}-\mathrm{T}_{1}} \\
(\mathrm{eV})\end{array}$ \\
\hline DONp $\alpha 1$ & 0.19 & 0.53 & 0.073 & 0.03 & 1.68 & 0.73 & 0.08 & 0.05 & 26.8 & $2.05 \pm 0.05$ & 2.68 & 0.63 \\
\hline DONp $\alpha 2\left(M_{\mathrm{W}} 1\right)$ & 0.31 & 0.46 & 0.25 & 0.20 & 3.34 & 0.45 & 0.24 & 0.26 & 35.3 & $1.75 \pm 0.05$ & 2.57 & 0.82 \\
\hline DONp $\alpha 2\left(M_{\mathrm{w}} 2\right)$ & 0.31 & 0.45 & 0.24 & 0.11 & 2.00 & 0.45 & 0.24 & 0.26 & 55.2 & $1.75 \pm 0.05$ & 2.58 & 0.83 \\
\hline DONp $\alpha 1 \mathrm{ThV}$ & 0.43 & 0.71 & 0.14 & 0.09 & 4.21 & 0.35 & 0.22 & 0.20 & 1.51 & $1.59 \pm 0.05$ & 2.48 & 0.89 \\
\hline $\operatorname{Naph}(\alpha 1)^{c}$ & 0.044 & 0.30 & 0.044 & & & $\approx 0$ & $0.9^{d}$ & & $20^{d}$ & 2.021 & 3.65 & 1.63 \\
\hline $\operatorname{Naph}(\alpha 2)^{c}$ & 0.14 & 0.36 & 0.12 & & & $\approx 0$ & $0.86^{b}$ & & $16^{b}$ & 1.932 & 3.44 & 1.51 \\
\hline
\end{tabular}

${ }^{a}$ Values obtained in MCH. ${ }^{b}$ Obtained in benzene. ${ }^{c}$ Data in $\mathrm{MCH}$ from ref 7. ${ }^{d}$ Values in dioxane. ${ }^{e}$ Values taken from the intersection between the room temperature absorption and fluorescence emission spectra. ${ }^{f} \phi_{\mathrm{IC}}=1-\left(\phi_{\mathrm{F}}+\phi_{\mathrm{T}}\right) .{ }^{g}$ Associated errors of $\pm 5 \% .{ }^{h}$ Associated error of \pm $15 \%$.

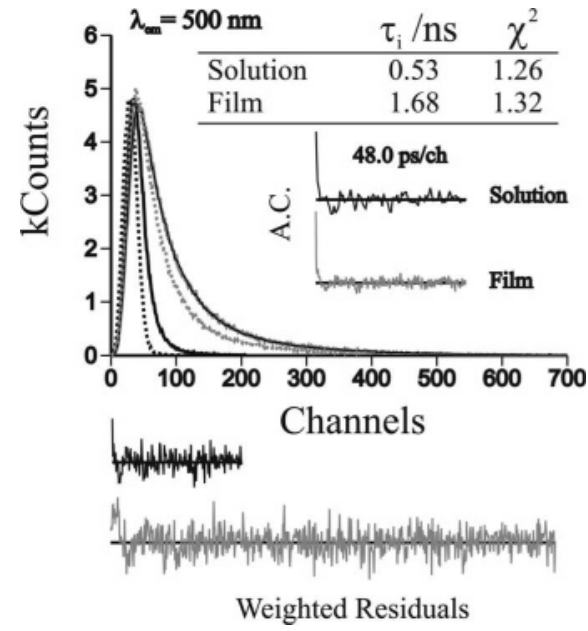

Figure 3. Fluorescence decay for DONp $\alpha 1$ in toluene solution at 293 $\mathrm{K}$ and in film. The presented fits are adjusted to monoexponential laws. For a better judgment of the quality of the fits, autocorrelation functions (ACs), weighted residuals and chi-square values $\left(\chi^{2}\right)$ are also presented as insets. The dashed lines in the decay represent the pulse instrumental response in solution and in the solid state.

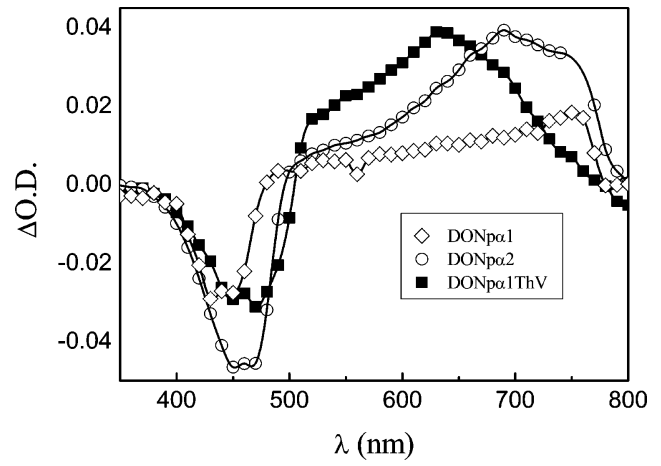

Figure 4. Transient triplet-triplet absorption spectra for the copolymers in toluene at room temperature. The $\mathrm{DONp} \alpha 2$ polymer presented is labeled with $M_{\mathrm{W}} 1$ (see Table 1, Scheme 1, and text for further details).

$k_{\mathrm{IC}}$ in DONp$\alpha 1 \mathrm{ThV}$, which is the dominant cause of the increase in the $\phi_{\mathrm{F}}$ value for this compound in comparison with DONp $\alpha 1$.

When the comparison is made between the polymers and the analogous oligomers, the 1-naphthyl(oligo)thiophenes, ${ }^{7,17}$ $\operatorname{Naph}(\alpha 1)$, and $\operatorname{Naph}(\alpha 2)$, the results are more dramatic. First, there is a clear red-shift of the absorption and emission maxima for all the polymers when compared with the oligomers (see Table 1). This indicates that even though small changes may arise from the presence of the dioctyl-substituted naphthalene in the polymer, there is additional involvement of more than one conjugation unit (one naphthalene and one or two thiophene units) in this system. It is also worth noting the large difference

\section{SCHEME 2}

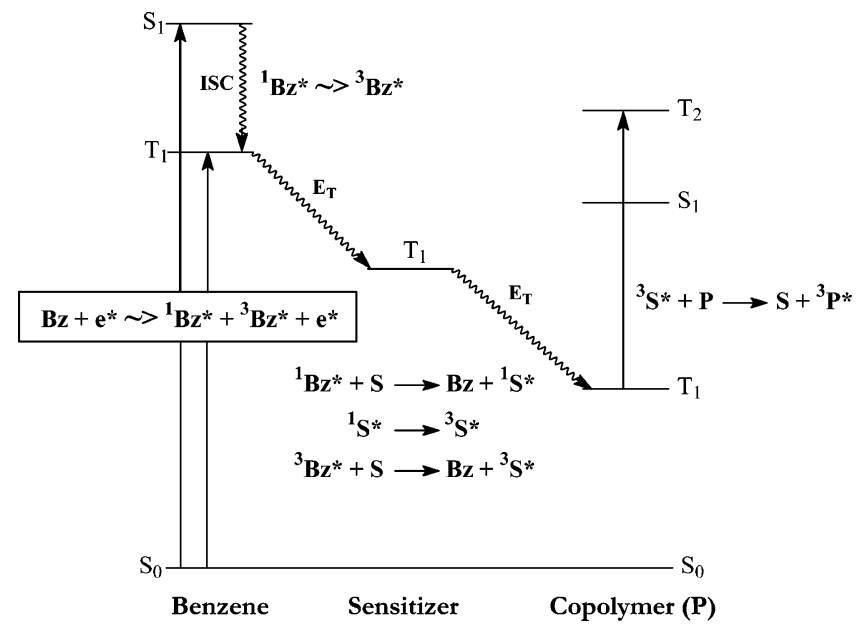

TABLE 3: Radiative $\left(k_{\mathrm{F}}\right)$ and Radiationless $\left(k_{\mathrm{NR}}, k_{\mathrm{ISC}}\right.$, and $k_{\text {IC }}$ ) Rate Constants for the Studied Copolymers in Toluene at Room Temperature (293 K), Low Temperature (77 K), and in Thin Films

\begin{tabular}{|c|c|c|c|c|}
\hline compound & $\begin{array}{c}k_{\mathrm{F}}\left(\mathrm{ns}^{-1}\right) \\
293 \mathrm{~K}^{b}\end{array}$ & $\begin{array}{c}\mathrm{k}_{\mathrm{NR}}\left(\mathrm{ns}^{-1}\right) \\
293 \mathrm{~K}^{c}\end{array}$ & $\begin{array}{c}\mathrm{k}_{\mathrm{IC}}\left(\mathrm{ns}^{-1}\right) \\
293 \mathrm{~K}^{d}\end{array}$ & $\begin{array}{c}\mathrm{k}_{\mathrm{ISC}}\left(\mathrm{ns}^{-1}\right) \\
293 \mathrm{~K}^{e}\end{array}$ \\
\hline DONp $\alpha 1$ & 0.358 & 1.53 & 1.38 & 0.151 \\
\hline DONp $\alpha 2\left(M_{\mathrm{W}} 1\right)$ & 0.674 & 1.50 & 0.98 & 0.521 \\
\hline DONp $\alpha 2\left(M_{\mathrm{W}} 2\right)$ & 0.688 & 1.53 & 1.00 & 0.533 \\
\hline DONp $\alpha 1 \mathrm{ThV}$ & 0.606 & 0.802 & 0.49 & 0.31 \\
\hline $\operatorname{Naph}(\alpha 1)^{a}$ & 0.147 & 3.19 & 0 & 3.2 \\
\hline $\operatorname{Naph}(\alpha 2)^{a}$ & 0.389 & 2.39 & 0 & 2.39 \\
\hline
\end{tabular}

between the Stokes Shift $\left(\Delta_{\mathrm{SS}}\right.$ in Table 1) of the oligomers relative to the polymers. With the polymers, this shift displays modest values $\left(\sim 2200 \mathrm{~cm}^{-1}\right)$, whereas with the oligomers the shift is much larger $\left(\sim 6900 \mathrm{~cm}^{-1}\right)$. This suggests that structural changes between the ground and first-excited singlet state are more significant with the oligomers than with the polymers. Additionally, with the polymer structural heterogeneity, energy migration, and planarization of different segments of the polymers may explain the observed difference.

With the fluorescence spectra of the polymers at room temperature and low temperature (Figure 2 and Table 1), there is only a slight red-shift upon going to $77 \mathrm{~K}$. This implies that there is basically no change in their structure with temperature, which is consistent with a relatively rigid structure involving the naphthalene and thiophene units (with the contribution of quinoid resonance structures in the excited state $)^{7,10}$ structure. However, this is not true with the absorption spectra of the polymers in which pronounced red-shifts and increasing struc- 
ture are observed upon going from room to low temperature (Figure 1 and Table 1). This can be attributed to an increase in the population of more planar conformers in the ground-state (relative to room temperature) with subsequent greater $\pi$-conjugation. ${ }^{7,10}$ The fluorescence quantum yields at low temperature are, in general, lower than those obtained at room temperature (see Table 2), which is in contrast with the constancy of this parameter (at both temperatures) observed for the parent Naph$(\alpha n s)^{7,17}$ and also other oligothiophenes. ${ }^{10,17}$ It is possible that some aggregation occurs at low temperature, as suggested by the small red-shift in emission.

In the solid state in a Zeonex matrix, it can be seen from Table 2 that, as with many other $\pi$-conjugated systems, the fluorescence quantum yield decreases relative to the room temperature solution value. This behavior is commonly attributed to the increase of the nonradiative decay channels. ${ }^{25,26}$ Again, aggregation may be important, and the red-shift observed in the absorption and emission spectra when going from solution to thin films may be associated with increasing intrachain and/ or interchain interaction generated in the solid state of the polymer. One additional observation concerns the large redshift in fluorescence for DONp $\alpha 2$ when going from solution to the solid state. This is similar to what was found with F8BT [poly(9,9-dioctylfluorene-alt-benzothiazole] and may indicate crystallization. ${ }^{27}$

Also note in Table 2 that the polymers have lower $S_{1} \sim \sim T_{1}$ intersystem-crossing yields compared with the parent Naph$(\alpha n)$, although the connection of the naphthalene and thiophene units is different for oligomers and polymers (1-naphthyl for the oligomers and 2,6-naphthylene for the copolymers). This may cause the differences in the intersystem crossing yields which can be attributed to the enhancement of the fluorescent deactivation channel due to the presence of the 1,5-dioctyloxynaphthalene groups in the copolymers. These apparently decrease the degree of charge character of the singlet state ${ }^{1} \Psi_{\mathrm{CT}}$ ) with a consequent decrease of the coupling between the charge-transfer (CT) state and the triplet state $\left({ }^{3} \Psi_{\mathrm{i}}\right)$. This decrease in intersystem-crossing with the copolymers indicates a route to enhancing fluorescence and decreasing triplet state formation in thiophene-containing copolymers. With the parent $\operatorname{Naph}(\alpha n)^{7}$ and the unsubstituted oligothiophenes $(\alpha n),{ }^{10,26}$ a decrease of $\phi_{\mathrm{T}}$ is observed with an increase in the number of thiophene units (and consequently the S-atoms). This means that the ISC in these oligomers cannot only be attributed to a classical spin orbit (SO) coupling effect, and it was interpreted as being due to a coupling mediated by $\mathrm{CT}$ mixing involving matrix elements of the type

$$
\left\langle{ }^{1} \Psi_{\mathrm{CT}}\left|\mathrm{H}^{\prime}\right|^{3} \Psi_{\mathrm{i}}\right\rangle
$$

where $\mathrm{H}^{\prime}$ is the $\mathrm{SO}$ (coupling) operator and contains the atomic SO coupling factor $\zeta$ for sulfur that was caused by a decrease in the overlap of the electron donor-electron acceptor molecular orbitals. ${ }^{7,10}$ It is relevant to this discussion that with the $\alpha$ and $\operatorname{Naph}(\alpha n)$ there was a negligible contribution of the internal conversion deactivation channel. This behavior is the opposite to that found with the polymers in which this is an important excited-state deactivation pathway (see Table 2). A significant contribution from internal conversion has been found with other thiophene oligomers ${ }^{26,28}$ and polymers. ${ }^{29}$ In the polymers, this was attributed to an efficient coupling promoted by the alkyl chains (present in the polymers), allowing the molecule to deactivate via the internal conversion channel. ${ }^{30}$ With the present systems, a similar effect is likely due to the dioctyloxy chains of the naphthalene units.

\section{SCHEME 3}

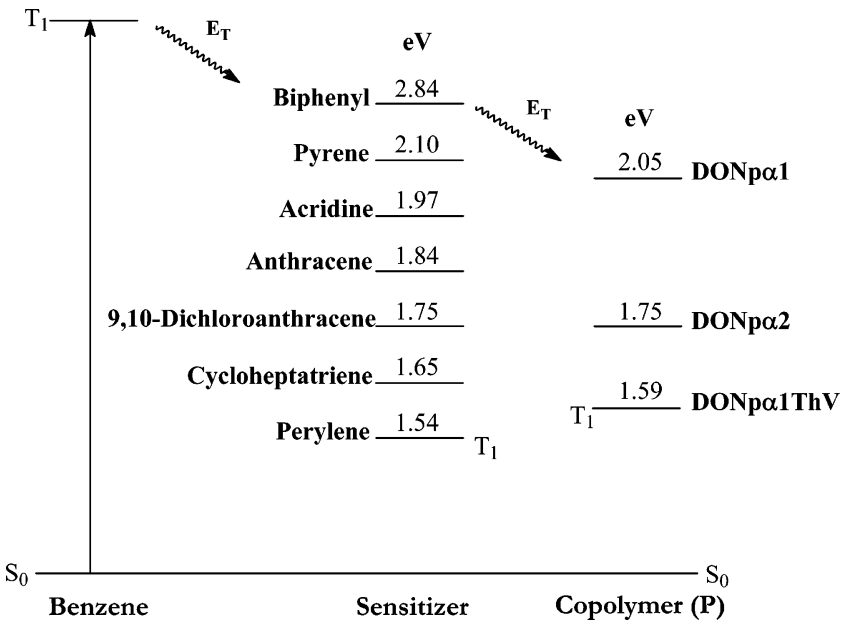

Considering the singlet oxygen yields for these polymers (see Table 2), these clearly resemble the triplet yield values showing efficient sensitization of molecular oxygen by the triplet state of the polymers which, besides providing support for the measured intersystem-crossing quantum yields, suggests that reaction with this may be an important pathway for degradation of devices produced with these polymers.

In the absence of phosphorescence, we have used the pulse radiolysis energy transfer method to obtain the triplet energy of the copolymers. Solutions of $\mathrm{P}$ were made up in benzene with sensitizers, $\mathrm{S}$, that have well-characterized $T_{1}$ states and different triplet energies.

In pulse radiolysis energy transfer measurements on degassed solutions of $\mathrm{P}$, detection of the triplet-triplet absorption indicates efficient sensitization and thus the $\mathrm{P}$ triplet state is below $\mathrm{S}$; when the energy of the acceptor (copolymer) lies above that of the sensitizer (i.e., when $\mathrm{E}_{\mathrm{T}}(\mathrm{S})<\mathrm{E}_{\mathrm{T}}(\mathrm{P})$ ) no induced triplet absorption is observed. For very accurate determination of the triplet energy of $\mathrm{P}$, an acceptor whose triplet energy lies very close to that of the sample is thus required. Using a wide range of triplet acceptors, energy transfer experiments have been performed on these copolymers in benzene, and we have obtained triplet energies for the copolymers under investigation, which range from 1.59 to $2.05 \mathrm{eV}$ (Scheme 3).

The singlet and triplet energies of the copolymers, shown in Table 2, are significantly lower than those of the correspondent $\mathrm{Naph}(\alpha \mathrm{n})$ oligomers indicating a lower HOMO-LUMO energy difference due to an extended $\pi$-delocalization. The triplet energy in $[\mathrm{Naph}(\alpha 1)](2.021 \mathrm{eV})$ and DONp $\alpha 1$ (2.05 eV) (see Table 2) is higher than in the corresponding fluorene-thiophenealternating copolymer ( $\left.\mathrm{PF} \alpha \mathrm{T}, 1.84 \mathrm{eV}^{31}\right)$ showing that the 2,6napthalene unit makes a weaker contribution to the conjugation unit than the 2,7-fluorene units.

The singlet-to-triplet energy splitting $\left(\Delta E_{\mathrm{S}_{1}}-{ }_{\mathrm{T}_{1}}\right)$ of the copolymers is similar to other conjugated polymers and copolymers, ${ }^{31,32}$ but is considerably lower than with the oligomers. However, although a larger energy gap in the latter case would suggest less efficient intersystem-crossing with the oligomers, the opposite is observed. This supports previous suggestions that intersystem-crossing has a significant CT character with the oligomers ${ }^{7,10}$ and suggests this may be more important than with the polymers.

From the rate constants presented in Table 3, it is clear that the radiationless pathways are dominant both in the oligomers $[\operatorname{Naph}(\alpha 1,2)]$ and polymers. However, with the oligomers $k_{\text {ISC }}$ is dominant, whereas in the polymers internal conversion is the 
main excited-state deactivation pathway. Furthermore, with the DONp $\alpha 1 \mathrm{ThV}$ polymer there is a decrease in the radiationless contribution, and the values for the $k_{\mathrm{F}}$ and $k_{\mathrm{NR}}$ rate constants are effectively identical.

With the copolymer DONpa2, samples with two different molecular weights and polydispersities were used (see Scheme 1). In general, the spectral and photophysical properties were very similar. The only significant difference occurred with the spectra in films in a Zeonex matrix in which more structure was observed with the lower molecular weight (DONp $\left.22 M_{\mathrm{W}} 1\right)$ polymer. This may be due to its better incorporation in the matrix.

\section{Conclusions}

We have investigated the photophysical and spectroscopic properties of new copolymers possessing alternating thiophene and naphthalene units. The inclusion of both naphthalene and thiophene groups in the conjugation unit was shown by both absorption and emission spectral studies. The polymers have improved luminescence yields in comparison with the analogous naphthalene $-\alpha$-oligothiophenes. However, in general, the excitedstate decay is dominated by nonradiative processes with internal conversion being particularly important. Although the energy separation between the lowest-excited singlet and triplet states is smaller than with the oligomers, intersystem-crossing is more prominent in the latter case. This supports the role of CT contributions to this process in the oligomers and suggests the decreased importance of these in the copolymers leading to decreased triplet state yields.

Acknowledgment. Financial support from FEDER and FCT (through projects POCI/QUI/55672/2004 and POCI/QUI/58291/ 2004) is acknowledged. J.P. acknowledges FCT for a Ph.D. grant (SFRH/BD/18876/2004). Pulse radiolysis experiments were carried out at the Free Radical Research Facility in the Synchrotron Radiation Department of the CLRC Daresbury Laboratory, Warrington, UK, with the support of the European Commission through the "Improving Human Potential" Transnational Access to Major Research Infrastructures Contract HPRI-CT-2002-00183. Drs. S. Navaratnam and R. Edge (FRRF, Daresbury Laboratory) are acknowledged for their excellent technical support in these.

Supporting Information Available: Synthesis and characterization of the copolymers together with the absorption and emission spectra for thin films obtained by direct spin-coating of a DONpa2 $\left(M_{\mathrm{W}} 2\right)$ toluene solution and blended with zeonex. This material is available free of charge via the Internet at http:// pubs.acs.org.

\section{References and Notes}

(1) Li, Y. N.; Wu, Y. L.; Liu, P.; Birau, M.; Pan, H. L.; Ong, B. S. Adv. Mater. 2006, 18, 3029.
(2) Majewski, L. A.; Kingsley, J. W.; Balocco, C.; Song, A. M. Appl. Phys. Lett. 200688. 4645 .

(3) Somanathan, N.; Radhakrishnan, S. Int. J. Mod. Phys. B 2005, 19,

(4) Perepichka, I. F.; Perepichka, D. F.; Meng, H.; Wudl, F. Adv. Mater. 2005, 17, 2281.

(5) Mucci, A.; Parenti, F.; Cagnoli, R.; Benassi, R.; Passalacqua, A.; Preti, L.; Schenetti, L. Macromolecules 2006, 39, 8293.

(6) Zhou, E. J.; Hou, J. H.; Yang, C. H.; Li, Y. F. J. Polym. Sci., Polym. Chem. Ed. 2006, 44, 2206.

(7) Seixas de Melo, J.; Silva, L. M.; Kuroda, M. J. Chem. Phys. 2001, 115,5625

(8) Blanchard, P.; Verlhac, P.; Michaux, L.; Frere, P.; Roncali, J. Chem.-Eur. J. 2006, 12, 1244 .

(9) Nehls, B. S.; Asawapirom, U.; Fuldner, S.; Preis, E.; Farrell, T.; Scherf, U. Adv. Funct. Mater. 2004, 14, 352.

(10) Becker, R. S.; Seixas de Melo, J.; Maçanita, A. L.; Elisei, F. J. Phys. Chem. 1996, 100, 18683

(11) Murov, S.; Charmichael, I.; Hug, G. L. Handbook of Photochemistry; Marcel Dekker: New York, 1993.

(12) Striker, G.; Subramaniam, V.; Seidel, C. A. M.; Volkmer, A. J. Phys. Chem. B 1999, 103, 8612

(13) Bensasson, R. V.; Land, E. J.; Truscott, T. G. Excited States and Free Radicals in Biology and Medicine; Oxford Science Publications: Oxford, 1993.

(14) Kumar, C. V.; Qin, L.; Das, P. K. J. Chem. Soc., Faraday Trans. 1 1984, 80, 783.

(15) Burrows, H. D.; Seixas de Melo, J.; Forster, M.; Guntner, R.; Scherf, U.; Monkman, A. P.; Navaratnam, S. Chem. Phys. Lett. 2004, 385, 105.

(16) Martinez, C. G.; Neumer, A.; Marti, C.; Nonell, S.; Braun, A. M.; Oliveros, E. Helv. Chim. Acta 2003, 86, 384.

(17) Pina, J.; Burrows, H. D.; Becker, R. S.; Dias, F. B.; Maçanita, A. L.; Seixas de Melo, J. J. Phys. Chem. B 2006, 110, 6499.

(18) Monkman, A. P.; Burrows, H. D.; Miguel, M. D.; Hamblett, I.; Navaratnam, S. Chem. Phys. Lett. 1999, 307, 303

(19) Burrows, H. D.; Seixas de Melo, J.; Serpa, C.; Arnaut, L. G.; Monkman, A. P.; Hamblett, I.; Navaratnam, S. J. Chem. Phys. 2001, 115, 9601.

(20) Karabunarliev, S.; Bittner, E. R. Phys. Rev. Lett. 2003, 90, 057402.

(21) Charas, A.; Morgado, J.; Martinho, J. M. G.; Alcacer, L.; Cacialli, F. Chem. Commun. 2001, 1216.

(22) Zhou, C. Z.; Wang, W. L.; Lin, K. K.; Chen, Z. K.; Lai, Y. H. Polymer 2004, 45, 2271.

(23) Kang, T. J.; Kim, J. Y.; Kim, K. J.; Lee, C.; Rhee, S. B. Synth Met. 1995, 69, 377.

(24) Turbiez, M.; Frere, P.; Roncali, J. Tetrahedron 2005, 61, 3045

(25) Nehls, B. S.; Galbrecht, F.; Bilge, A.; Brauer, D. J.; Lehmann, C. W.; Scherf, U.; Farrell, T. Org. Biomol. Chem. 2005, 3, 3213.

(26) Pina, J.; Seixas de Melo, J.; Burrows, H. D.; Bilge, A.; Farrell, T.; Forster, M.; Scherf, U. J. Phys. Chem. B 2006, 110, 15100.

(27) Grell, M.; Redecker, M.; Whitehead, K. S.; Bradley, D. D. C.; Inbasekaran, M.; Woo, E. P.; Wu, W. Liq. Cryst. 1999, 26, 1403.

(28) Anni, M.; Della Sala, F.; Raganato, M. F.; Fabiano, E.; Lattante, S.; Cingolani, R.; Gigli, G.; Barbarella, G.; Favaretto, L.; Gorling, A. J. Phys. Chem. B 2005, 109, 6004

(29) Huang, Y. F.; Chen, H. L.; Ting, J. W.; Liao, C. S.; Larsen, R. W.; Fann, W. J. Phys. Chem. B 2004, 108, 9619

(30) Seixas de Melo, J.; Burrows, H. D.; Svensson, M.; Andersson, M. R.; Monkman, A. P. J. Chem. Phys. 2003, 118, 1550.

(31) Fonseca, S. M.; Pina, J.; Arnaut, L. G.; Seixas de Melo, J.; Burrows, H. D.; Chattopadhyay, N.; Alcácer, L.; Charas, A.; Morgado, J.; Monkman, A. P.; Asawapirom, U.; Scherf, U.; Edge, R.; Navaratnam, S. J. Phys. Chem. $B$ 2006, 110,8278 .

(32) Monkman, A. P.; Burrows, H. D.; Hartwell, L. J.; Horsburgh, L. E.; Hamblett, I.; Navaratnam, S. Phys. Rev. Lett. 2001, 86, 1358 